\title{
Unit Squares Intersecting All Secants of a Square
}

\author{
Pavel Valtr* \\ Department of Applied Mathematics, Charles University, \\ Malostranské nám. 25, 11800 Praha 1, Czech Republic \\ valtr@espguk11.bitnet \\ and \\ Graduiertenkolleg “Algorithmische Diskrete Mathematik", Fachbereich Mathematik, \\ Freie Universität Berlin, Arnimallee 2-6, 1000 Berlin 33, Germany \\ valtr@math.fu-berlin.de
}

\begin{abstract}
Let $S$ be a square of side length $s>0$. We construct, for any sufficiently large $s$, a set of less than $1.994 \mathrm{~s}$ closed unit squares whose sides are parallel to those of $S$ such that any straight line intersecting $S$ intersects at least one square of $S$. It disproves $\mathbf{L}$. Fejes Tóth's conjecture that, for integral $s$, there is no such configuration of less than $2 s-1$ unit squares.
\end{abstract}

\section{Introduction}

The following conjecture is due to Fejes Tóth [FT], [MP]: "Given n points in the unit square, there exists a line intersecting the unit square, which has $L_{\infty}$-distance at least $1 /(n+1)$ from each point."

This can obviously be restated as follows: let $S$ denote an axis parallel square of side length $s$ and let $\mathscr{U}=\left\{U_{1}, \ldots, U_{t}\right\}$ be any collection of axis parallel unit squares lying in $S$. We say that $\mathscr{U}$ is a line cover of $S$ if every line which intersects $S$ also intersects a square of $\mathscr{U}$. Let $\tau(s)$ denote the smallest $t \in \mathbb{N}$ such that there exists a line cover of $S$ of size $t$. Then Fejes Tóth's conjecture states that $\tau(s) \geq 2 s-1$ (the configuration in Fig. 1 gives $\tau(s) \leq 2 s-1$ for $s$ odd). We disprove this conjecture by a construction which shows $\tau(s) \leq 1.994 s$ for $s$ sufficiently large.

Several years ago Bárány and Füredi studied the following version of this problem. Let $\tau^{\prime}(s)$ be defined in the same way as $\tau(s)$, except that we want to cover

\footnotetext{
* Supported by "Deutsche Forschungsgemeinschaft", Grant We 1265/2-1.
} 


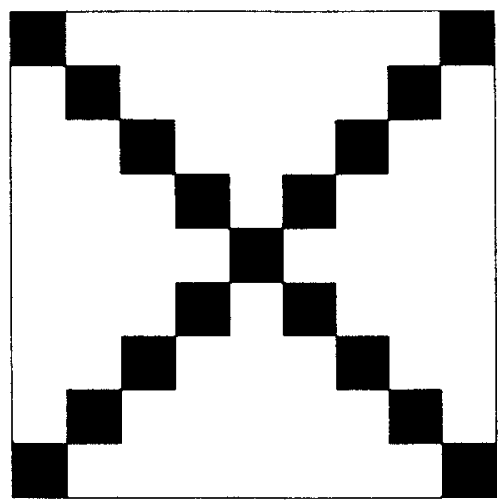

Fig. 1. Line cover of size $2 n-1$.

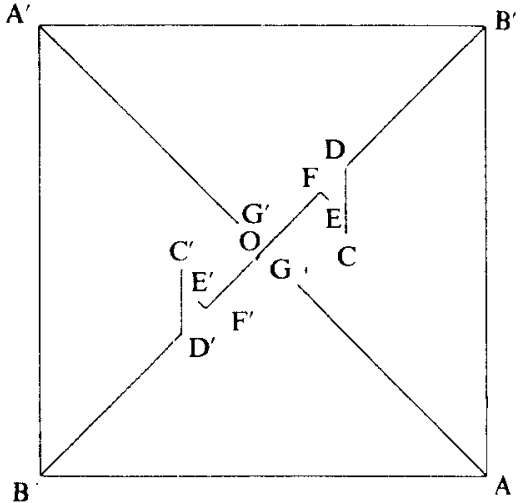

Fig. 2. Points in the square $S$.

only those lines which are parallel to one of the sides of $S$ or to one of the diagonals of $S$. So we restrict our attention to lines of four directions. Bárány and Füredi $[\mathrm{BF}]$ (see also $[\mathrm{KW}]$ ) proved that $\tau^{\prime}(s) \geq \frac{4}{3} s-\frac{1}{3}$. On the other hand, Kern and Wanka [KW] constructed sets which give an upper bound of $\tau^{\prime}(s) \leq \frac{4}{3} s+\mathcal{O}(1)$.

\section{Construction}

Consider first an axis parallel square $S$ with side length 2 centered at the origin. We define points $A, B, C, D, E, F, G, O$ by the following relations (see Fig. 2; for technical reasons ratios of distances on it are changed):

$O=[0,0], \quad A=[1,-1], \quad B=[1,1]$,

$O, F, D, B$ are collinear,

$O, G, A$ are collinear,

$C D$ is parallel to $A B, \quad E F$ is parallel to $O A$,

$$
\begin{aligned}
& |O D|=\frac{1}{10}|O B|, \quad|F D|=\frac{1}{100}|O B|, \quad|F E|=\frac{1}{1000}|O B|, \\
& |O G|=\frac{1}{100}|O A|, \quad|C D|=\frac{11}{1000}|A B|=0.022 .
\end{aligned}
$$

The points $A^{\prime}, B^{\prime}, C^{\prime}, D^{\prime}, E^{\prime}, F^{\prime}, G^{\prime}$ are defined as the images of the points $A$, $B, C, D, E, F, G$ obtained by reflection about the origin.

Here is a technical lemma:

Lemma 1. Each line intersecting the square $S$ intersects some of the line segments $A G, G^{\prime} A^{\prime}, B^{\prime} D^{\prime}, D^{\prime} C^{\prime}, E^{\prime} F^{\prime}, F^{\prime} F, F E, C D$, and $D B$. 
Proof. Due to symmetry the lemma follows from the following three facts:

1. The point $C$ lies below the line $A F$.

2. The point $G$ lies above the line $B^{\prime} C$.

3. The point $E$ lies on the line $D G$.

Now we scale the square $S$ and all distances among points in it by the factor $s / 2$ in order that the square $S$ has the side length $s$, and construct our set as follows. We take unit squares on the line segments $A G, G^{\prime} A^{\prime}, B^{\prime} D^{\prime}, E^{\prime} F^{\prime}, F^{\prime} F, F E, D B$ of slope \pm 1 so that two consecutive squares always have one common point (vertex) similarly as the squares on the diagonals in Fig. 1. On each of these seven line segments we take the minimum number of unit squares so that they cover the line segment. Further, we take unit squares with centers on the vertical line segments $C^{\prime} D^{\prime}$ and $D C$ so that two consecutive squares have a distance 0.8 (see Fig. 3 ). On each of these two line segments we take the minimum number of unit squares so that their convex hull covers the line segment. We denote the obtained set of unit squares by $\mathscr{U}$.

Theorem 2. For any sufficiently large s there is a set $\mathscr{U}$ of less than 1.994s axis parallel unit squares which is a line cover of $S$.

Proof. $\mathscr{U}$ is constructed above so that it is a line cover of $S$. The number of squares of the set $\mathscr{U}$ is $0.99 s+\mathcal{O}(1)$ on each of the diagonals, $0.0005 s+\mathcal{O}(1)$ on both the line segments $E^{\prime} F^{\prime}$ and $E F$, and $0.011 \cdot(1 / 1.8) s+\mathcal{O}(1)$ on both the line
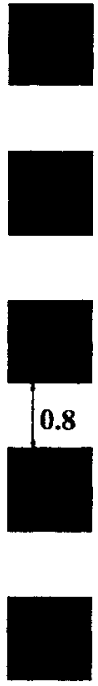

Fig. 3. String of unit squares used in the construction.

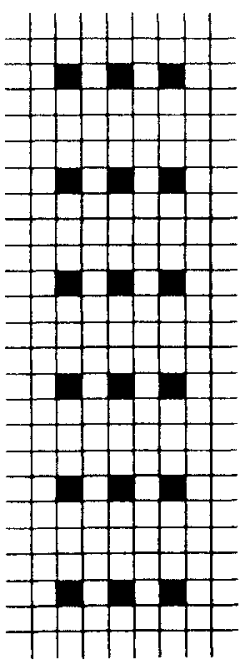

Fig. 4. String of unit squares embeddable to the chess board. 
segments $C^{\prime} D^{\prime}$ and $C D$. Altogether, there are

$$
2(0.99 s+0.0005 s+0.011 \cdot(1 / 1.8) s)+\mathcal{O O}(1)=1.9932 \ldots s+\mathcal{O}(1)
$$

squares in $\mathscr{U}$. Theorem 2 immediately follows.

\section{Remarks}

1. The constant 1.994 in Theorem 2 can obviously still be improved. It is better to consider some curves instead of the line segments in Fig. 2, and on them put unit squares with "continuously" changing intervals among them. In this way we can get a constant smaller than 1.99. On the other hand, Bárány and Füredi $[\mathrm{BF}]$ showed the lower bound $\tau(s)>1.43 s+\mathcal{O}(1)$.

2. Consider Fejes Tóth's problem with the following restriction. The number $s$ is integral and the set $\mathscr{U}$ may contain only integral unit squares, i.e., unit squares whose vertices have integral coordinates. In other words, we are choosing squares of the chess board $S=s \times s$. The solution in Fig. 1 is again not optimal:

Theorem 3. For any sufficiently large $s$ there is a set $\mathscr{U}$ of less than 1.998 s integral unit squares, which is a line cover of $S$.

Proof. We proceed similarly as in Section 2 with the exception that along the vertical line segments $C^{\prime} D^{\prime}$ and $C D$ we place squares as shown in Fig. 4 instead of the string of squares in Fig. 3. Then the set $\mathscr{U}$ contains

$$
2\left(0.99 s+0.0005 s+0.011 \cdot \frac{3}{4} s\right)+\mathcal{O}(1)=1.9975 s+\mathcal{O}(1)
$$

squares.

3. Bárány and Füredi $[B F]$ note that Erdbs asked what is the minimum size of a line cover $\mathscr{U}$ if $\mathscr{U}$ contains only axis parallel unit squares outside $S$. They mention a trivial upper bound $3 s+\mathcal{O}(1)$. This bound is obtained by a configuration of unit squares placed tightly one by one along three sides of the square $S$. If we place squares along one of the three sides with appropriate distances among them we get a better upper bound $(2+\ln 2) s+\mathcal{O}(1)<2.7 s+\mathcal{O}(1)$. There is also a similar configuration of $(2+\ln 2) s+\mathcal{O}(\sqrt{s})$ integral unit squares outside $S$ forming a line cover of $S$.

4. There is another question about the minimum size of a line cover $\mathscr{U}$ if $\mathscr{U}$ contains arbitrary unit squares (not necessarily axis parallel). This question is related to a question about the minimum total length of curves inside a unit square which intersect all lines intersecting the unit square. Four line segments of total length $\frac{1}{2} \sqrt{2}(2+\sqrt{3})=2.638$.. are conjectured to form an optimal solution 


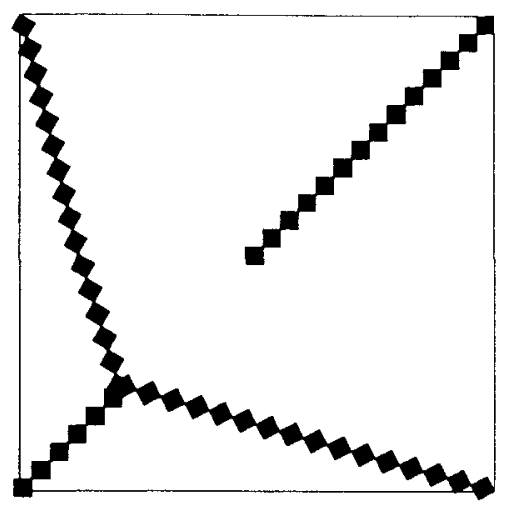

Fig. 5. Line cover of a square by unit squares.

(see [B]). If we place unit squares along these four line segments we get a line cover of $S$ of size $\frac{1}{2}(2+\sqrt{3}) s+\mathcal{O}(1)=1.866 \ldots s+\mathcal{O}(1)$ (see Fig. 5). This might be an optimal solution to the problem.

\section{References}

[B] K. A. Brakke, The opaque cube problem, Amer. Math. Monthly 99(9) (1992), 866-871.

[BF] I. Bárány and Z. Füredi, Covering all secants of a square, Proceedings of the Colloquia Mathematica Societatis János Bolyai, Siofok, Hungary, 1985 (in honor of L. Fejes Tóth).

[FT] L. Fejes Tóth, Remarks on dual of Tarski's plank problem, Mat. Lapok 25 (1974), 13-20.

[KW] W. Kern and A. Wanka, On a problem about covering lines by squares, Discrete Comput. Geom. 5 (1990), 77-82.

[MP] W. O. Moser and J. Pach, Research Problems in Discrete Geometry, Problem 84, Montréal 1985, mimeographed.

Received June 12, 1992, and in revised form October 13, 1992. 